\title{
Quenching of luminescence in semiconductors by charged defects
}

\author{
P W Tasker and A M Stoneham \\ Theoretical Physics Division, AERE, Harwell, Didcot, Oxon OX11 0RA, UK
}

Received 27 June 1977

\begin{abstract}
Charged point defects and dislocations create internal electric fields that can prevent the binding of carriers to luminescent centres such as $\mathrm{N}$ in GaP. This paper calculates the distribution of fields present in a semiconductor and its effect on the lifetime of an electron trapped at a localised impurity. The results show a decrease in the fraction of effective luminescent centres with increasing concentration of ionised donors and acceptors Both unscreened dislocations and those with a compensting atmosphere of oppositely charged point defects are considered and the calculations show dark regions around them, as observed.
\end{abstract}

\section{Introduction}

Light-emitting diodes are of considerable commercial importance and are usually constructed from III-V compound semiconductors, in particular GaP and GaAs ${ }_{1-x} \mathrm{P}_{x}$. The quantum efficiency of these devices is, however, low owing to competing nonradiative pathways to recombination. The luminescence is produced by the injection of minority carriers, for example across a $\mathrm{p}-\mathrm{n}$ junction under an applied voltage (electroluminescence), and is usually associated with the localised states of impurities and dopants rather than a direct interband transition. Indeed, in $\mathrm{GaP}$, the direct transition is particularly unlikely since the bandgap is indirect. Most of the commercial devices emit red light, but there is considerable interest in devices that emit green light since the eye is many times more sensitive to this end of the spectrum. The energy of green light is close to the bandgap and so the states of any impurities involved must be shallow and this leads to very low efficiencies (see e.g., Moss et al 1973). Much of the current interest has centred on nitrogen substituted $\mathrm{GaP}$ since nitrogen impurities strongly enhance the green luminescence (see e.g., Dean 1976). Nitrogen is an isoelectronic impurity that replaces a phosphorus atom in GaP. It has been shown to give a bound electronic state that has been much studied both theoretically and experimentally (Thomas and Hopfield 1966, Faulkner 1968, Czaja 1971, Stoneham 1975) and it is estimated that this state lies $0.008 \mathrm{eV}$ below the conduction band.

Dislocations in the crystal structure are known to reduce the luminescent efficiency and there have been studies of the decrease of efficiency with increasing dislocation density. (Suzuki and Matsumoto 1975, Kuijpers et al 1975, Harding et al 1976). Microscopic studies of crystals that have been etched to reveal the dislocations have shown dark regions of little or no luminescence, a few $\mu \mathrm{m}$ wide around each dislocation in $\mathrm{GaP}$ and other semiconductors (Heinke 1974, Queisser 1976, Titschmarsh et al 1976, 
Kishino et al 1976, Iwamoto and Kasami 1976, Werkhoven et al 1976). Different types of dislocations (e.g. screw, edge or mixed) all appear to produce a similar effect (Titschmarsh et al 1976) and there has been much speculation concerning the mechanism of the dislocation induced quenching of luminescence.

In this paper we propose a mechanism for the quenching of luminescence by internal electric fields caused by the presence of charged dislocations and point defects. These fields are shown to be capable of preventing the binding of a carrier in a shallow state of an impurity or dopant and thus preventing luminescence from this centre. This mechanism can produce the dark regions around dislocations as observed and reduces the efficiency of the material by increasing the luminescence lifetime. The non-radiative, recombination processes that compete with luminescent processes are not considered here. The calculation consists of two parts: the influence of an electric field on a carrier in a localised bound state, and the distribution of electric fields in the crystal.

\section{Ionisation of impurity states}

An electric field can cause the bound state of a potential well to be degenerate with the continuum elsewhere, so that a trapped electron can escape by tunnelling through the intervening barrier. Problems of this sort are usually solved using the WKB method. But if the electric field is uniform, i.e. the potential energy is given by:

$$
U(x)=-|e| F x
$$

where $F$ is the electric field strength, the wavefunction outside the well can be expressed exactly in Airy functions. The tunnelling from a spherical potential well has been solved by Franz (1952) who assumed only that the electric field varied sufficiently slowly over the diameter of the well for the unperturbed wavefunction to be valid inside. This is a good approximation for the electric fields considered here. Franz obtains for the lifetime of the electron in the well, $\tau$,

$$
1 / \tau=\frac{k_{0}^{2}}{k_{0}^{2}+\chi^{2}} \frac{\exp (2 \chi R)}{(1+\chi R)} \frac{|e| F}{2} \frac{1}{\sqrt{\left(2 m^{*} \Delta E\right)}} \exp \left(-\frac{4}{3} \frac{\sqrt{\left(2 m^{*}\right)}}{\hbar|e| F} \Delta E^{3 / 2}\right),
$$

where $\Delta E$ is the binding energy of the electron in the well, $m *$ is the effective mass of the electron in the conduction band, $R$ is the radius of the well and $k_{0}$ and $\chi$ are given by:

$$
\hbar^{2} \chi^{2}=2 m^{*} \Delta E ; \quad \hbar^{2} k_{0}^{2}=2 m^{*}(V-\Delta E),
$$

where $V$ is the depth of the well.

The dimensions of the well, $V$ and $R$, are chosen to give the required binding energy $\Delta E$. The groundstate of the well is given by:

$$
\tan k_{0} R=-k_{0} / x
$$

The choice of well dimensions is not unique but the first two fractions in equation (2) are close to unity for small $\Delta E$ and vary only slightly with $R$ and $V$. The expression for the lifetime, therefore, does not depend strongly on the detailed shape of the potential and, indeed, almost all field emission theories lead to an expression of the form

$$
1 / \tau=A(|e| F)^{n} \exp \left(-\frac{B}{|e| F} \Delta E^{3 / 2}\right)
$$


where $A, B$ and $n$ depend on the type of process being considered (Duke 1969). Since the tunnelling rate is only weakly dependent on the details of the potential it is reasonable to represent the potential of the isoelectronic defect by a simple spherical well.

In deriving this expression and applying it to field emission in solids the influence of the crystal lattice on the electron's wavefunction has not been directly included. However, it is implicitly considered since the experimental effective mass for the electron in the appropriate semiconductor will be used in equation (2). The use of the unperturbed wavefunction inside the well limits the strength of the field for which the expression is valid. The approximation is found to be still valid when the lifetime of the electron in the well is equal to the radiative lifetime and only breaks down for much shorter lifetimes when we may consider the ionisation to be immediate (Franz 1952).

\section{Distribution of electric fields}

The presence of charged defects in crystals lead to random electric fields which may be observed by their effect on the shapes of resonance lines (Mims and Gillen 1966, Stoneham 1969, 1974) and the radiative decay of colour centres (Bennett and Stoneham 1972). We consider first the effect of charged point defects, for example, ionised donors and accepters, and calculate the probability of an electric field $\boldsymbol{F}$ at any point in the crystal. If the point charges are assumed to be randomly distributed the Holtsmark distribution describes the electric fields (Chandrasekhar 1943). The magnitude of the field irrespective of direction is given by

$$
P(\beta)=\frac{2}{\pi \beta} \int_{0}^{\infty} \mathrm{d} x x \sin x \exp \left[-(x / \beta)^{3 / 2}\right]
$$

where $\beta=F / Q$ and $Q$ is the characteristic field dependent on the density of point charges.

$$
Q=\frac{2 \pi}{\epsilon}\left(\sum_{\alpha} \frac{4}{15} \rho_{\alpha}^{0}\left|Z_{\alpha} e\right|^{3 / 2}\right)^{2 / 3}
$$

$\epsilon$ is the static dielectric constant and $\rho_{\alpha}^{0}$ is the number of defects per unit volume with charge $Z_{z}|e|$ relative to the perfect lattice. Since this distribution goes through a maximum at about 1.60 quite substantial fields may be experienced in crystals without dislocations, particularly at high doping levels.

We now consider the introduction of a dislocation which is electrically charged. In a binary and partly ionic semiconductor such as GaP, this charge is associated with jogs and bound vacancies (Whitworth 1975). The dislocation will be represented as a charged line with $Z^{\prime}|e|$ as the charge per unit length, the electric field a distance $r$ away is then given by

$$
\boldsymbol{F}_{\mathrm{o}}=\frac{2 Z^{\prime}|e|}{\epsilon r} \frac{\boldsymbol{r}}{r}
$$

If this dislocation has been freshly introduced by, for example, straining the crystal, the position of the point defects will be uncorrelated. We require the distribution of fields of magnitude $|F|$ where $\boldsymbol{F}=\boldsymbol{F}_{1}+\boldsymbol{F}_{0}$ and $\boldsymbol{F}_{1}$ is the field due to the distribution of point defects. Since at any point $r$ from the dislocation we are summing a constant field $\boldsymbol{F}_{0}$ and a distribution of fields $F_{1}$ the probability of there being a field $F$ is:

$$
P(\boldsymbol{F})=P\left(\boldsymbol{F}_{1}\right) \quad \text { where } \boldsymbol{F}_{1}=\boldsymbol{F}-\boldsymbol{F}_{0}
$$


and

$$
P\left(\boldsymbol{F}_{1}\right)=\left(1 / 2 \pi^{2} \beta_{1} Q^{3}\right) \int_{0}^{\infty} \mathrm{d} x \exp \left(-x^{3 / 2}\right) \sin \left(x \beta_{1}\right) x
$$

where

$$
\begin{aligned}
& \beta_{1}=\mid F_{1} / / Q \quad \text { (Chandrasekhar 1943) } \\
& P(|F|)=2 \pi|F|^{2} \int_{0}^{\pi} P(\boldsymbol{F}) \sin \theta \mathrm{d} \theta
\end{aligned}
$$

and

$$
\beta_{1}=\left(\beta^{2}+\beta_{0}^{2}-2 \beta \beta_{0} \cos \theta\right)^{1 / 2}
$$

where

$$
\begin{aligned}
& \beta_{0}=\left|F_{0}\right| / Q \quad \text { and } \quad \beta=|F| / Q \\
& P(\beta)=\frac{2 \beta}{\beta_{0} \pi} \int_{0}^{\infty} \mathrm{d} x \sin (x \beta) \sin \left(x \beta_{0}\right) \exp \left(-x^{3 / 2}\right) .
\end{aligned}
$$

Equation (13) gives the modified Holtsmark distribution for fields of magnitude $|F|=\beta Q$. It is implicitly dependent on $r$ since $\beta_{0}$ is the field due to the dislocation, and as $\beta_{0} \rightarrow 0$ it naturally becomes equal to the Holtsmark distribution in equation (6).

In the case of a grown-in dislocation the point defects will be correlated in position and so form a compensating charge cloud around it. The increasing density of charged point defects at positions near to the dislocation leads to a net electric field that opposes the field of the dislocation and thus leads to screening. The charge is usually treated as a continuum and the resulting electric field calculated from Poisson's equation (Whitworth 1972, Neubert 1974). Unfortunately analytical solutions may be obtained only in certain unrealistic limits (Eshelby et al 1958) so we have preferred to use a Debye-Hückel form for the screened electric field.

$$
\boldsymbol{F}_{0}=\left(2 Z^{\prime}|e| / \epsilon r\right)(\boldsymbol{r} / r) \exp \left(-r / R_{\mathrm{s}}\right)
$$

and $R_{\mathrm{s}}$, the screening radius is given by:

$$
R_{\mathrm{s}}^{-2}=\frac{4 \pi e^{2}}{\epsilon k T} \sum_{\alpha} Z_{\alpha}^{2} \rho_{\alpha}^{0}
$$

for point defëcts of charge $Z_{\alpha}|e|$ and mean density $\rho_{\alpha}^{0}$. This assumes a thermal equilibrium with $T$ as the equilibrium temperature. Equation (14) is not a solution of Poisson's equation in this case but approximates such a solution. It has previously been used in a calculation of line broadening by dislocations (Stoneham 1974).

We have described the effect of the point defects considered as providing a continuum charge cloud around the dislocation whereas they are, of course, individual point charges. The Holtsmark distributions (equations (6) and (13)) show that this leads to a significant probability of high electric fields existing at any point in the crystal owing to the proximity of charged point defects. This distribution depends on the density of point defects which increases near the dislocation and so the characteristic field $Q$ becomes a function of distance from the dislocation.

$$
Q(r)=(2 \pi / \epsilon)\left(\sum_{\alpha} \frac{4}{15} \rho_{\alpha}(r) \mid Z_{\alpha} e^{3 / 2}\right)^{2 / 3}
$$


and the density of point defects in thermal equilibrium is given by:

$$
\rho_{\alpha}(r)=\rho_{\alpha}^{0} \exp [\Phi(r) / k T]
$$

$\rho_{\alpha}^{0}$ is the density of point defects far from the dislocation, which at low dislocation density is equal to the mean density of point defects. For an electric field given by equation (14), the potential $\Phi(r)$ is

$$
\Phi(r)=\left(2 Z^{\prime} Z_{\alpha} e^{2} / \epsilon\right) E_{1}\left(r / R_{\mathrm{s}}\right)
$$

where $E_{1}(Z)$ is the exponential integral (Abramowitz and Stegun 1965). Summing the two sources of electric field gives the final distribution as in equation (13). The derivation of the Holtsmark function assumes a random distribution of point charges whereas this is no longer the case. We have, however, separately included the average effect of the increase in defect density as we approach the dislocation and the highest fields are due only to nearest neighbours (Chandrasekhar 1943) for which the local density (equation (17)) is valid. In calculating the distribution of fields due to a screened dislocation, equations (14) and (15) give the screened field due to the dislocation with charge cloud and equations (17) and (18) give the density of point defects as a function of distance from the dislocation. Equations (16) and (13) then give the final distribution for a particular distance $r$ from the dislocation.

\section{Quenching of luminescence}

In $\S \S 2$ and 3 we have shown how to calculate the internal electric field at any point in the crystal and its effect on an electron trapped at a luminescent centre. When the lifetime of the electron in the well becomes very short compared with the radiative lifetime, no radiation will be emitted. If however the lifetime is long then radiation may be emitted, the quantum efficiency depending on the competing non-radiative processes and thermal ionisation. We shall calculate the fraction of luminescent centres which can trap an electron for times that are long compared with the radiative lifetimes, i.e., centres in low field positions, and define this as the radiative fraction, $f_{\mathrm{r}}$. Figure 1 shows the lifetime of the electron in the well with respect to tunnelling as a function of field strength calculated from equation (2). We have used a binding energy of $0.01 \mathrm{eV}$, dielectric constant $\epsilon=11.0$ and an effective mass for the electron in the conduction band $m^{*}=0 \cdot 2 m_{0}$ where $m_{0}$

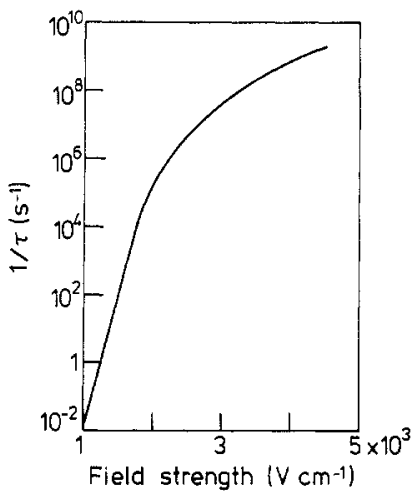

Figure 1. Lifetime of electron trapped in well versus electric field strength. Binding energy of electron $=0.01 \mathrm{eV}$. 
is the free electron mass. This approximates the behaviour of the $\mathrm{N}$ centre in GaP. Similar plots can be obtained for other binding energies with a shift to higher field strengths for deeper traps. It can be seen that the lifetime varies very rapidly with field strength and so we can define a critical field, $F_{\mathrm{c}}$, at which the lifetime with respect to tunnelling is equal to the radiative lifetime. A small change in $F_{c}$ will lead to almost either no radiation or no tunnelling. The probability of a radiative centre being in a position of lower field than $F_{\mathrm{c}}$ is the radiative fraction $f_{\mathrm{r}}$ and given by:

$$
f_{\mathrm{r}}=\int_{0}^{F \mathrm{c}} P(\beta) / Q \mathrm{~d} F .
$$

The radiative lifetime is required for the calculation of the critical field, and depends on the mechanism for the emission of radiation that is assumed. The radiative process from an isoelectronic centre such as $\mathrm{N}$ is assumed to be a sequential capture of electron, then hole to form an exciton which relaxes with the emission of radiation. For an electron trapped at a centre the competing radiative lifetime, $\tau_{\mathrm{r}}$, is then:

$$
\tau_{\mathrm{r}}=\tau_{\mathrm{hc}}+\tau_{\mathrm{se}}
$$

where $\tau_{\mathrm{hc}}$ is the lifetime for hole capture and $\tau_{\mathrm{se}}$ is the spontaneous emission lifetime. When there is an abundance of holes the spontaneous emission rate will be rate determining but, when there are few holes, for example at very low temperatures, the hole capture rate may be more important. The hole capture lifetime may be written as

$$
1 / \tau_{\mathrm{he}}=\sigma\langle v\rangle \rho_{\mathrm{h}}
$$

where $\sigma$ is the capture cross section, $\langle v\rangle$ the mean velocity estimated by assuming the energy to be $\frac{3}{2} k T$ and $\rho_{\mathrm{h}}$ is the hole density. The spontaneous emission rate can be written as (Stoneham 1975)

$$
1 / \tau_{\mathrm{se}}=\left(2 e^{2} / m^{*} c\right)(\omega / c)^{2} f
$$

where $\omega$ is the frequency of the radiation emitted and $f$ the oscillator strength. Taking the wavelength of light emitted as $560 \mathrm{~nm}$, green radiation from $\mathrm{GaP}$, and the oscillator strength for this system as 0.1 (Cuthbert and Thomas 1967) the radiative lifetime is $3.7 \times 10^{-7} \mathrm{~s}$. Figure 2 shows the critical field, $F_{\mathrm{c}}$, as a function of binding energy of the electron in the well assuming this value for the radiative lifetime.

In calculating the dependence of the radiative fraction on the concentration of charged point defects, $\tau_{\mathrm{hc}}$ has been included in the estimation of $\tau_{\mathrm{r}}$. The density of holes $\rho_{\mathrm{h}}$ is approximately equal to the density of charged acceptors present and this is of the same order of magnitude as the total density of point charge species. This is the case in most $\mathrm{n}$-type as well as p-type semiconductors. The capture cross-section is difficult to calculate with any reliability (Stoneham 1975) and experimental data are limited. Since the ionised $\mathrm{N}$ centre presents a coulomb attraction to the hole the capture cross-section may be expected to be insensitive to the centre and dependent mainly on the host. We have used a very low value of $1 \AA^{2}$ to maximise the observed effect, although a low value for the coulomb potential might be inferred from the very long bulk lifetimes observed in GaP. (Opdorp et al 1977). In any case, the low value will overestimate the effect, which will be shown to be negligible in the region of interest. Although the inclusion of $\tau_{\mathrm{hc}}$ effects the radiative lifetime it has only a small effect on the critical field for the hole densities present. Figure 3 shows the radiative fraction of luminescent centres as a function of concentration of point charges. As the concentration of point charges increases, so does 


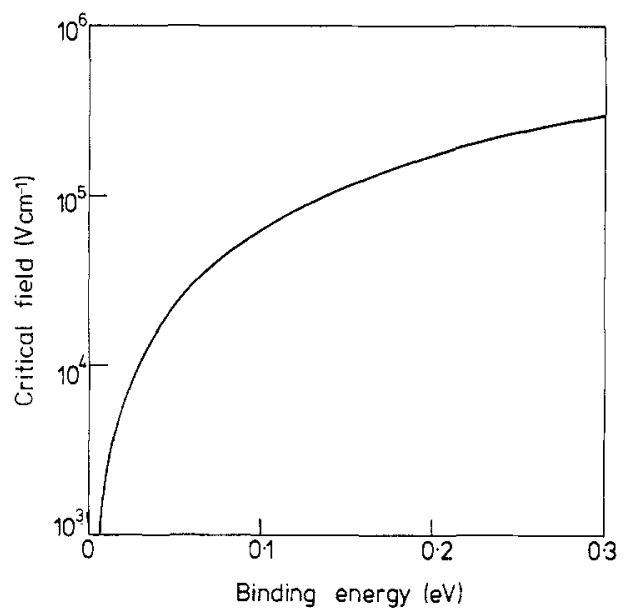

Figure 2. Critical field for electron tunnelling from well versus the binding energy of the electron.

the average internal electric field. This decreases the radiative fraction; at a concentration of $10^{18} \mathrm{~cm}^{-3}$, the radiative fraction is $\sim 10^{-3}$. Thus the effective concentration of nitrogen centres is reduced with increasing doping level and this should be included in recombination kinetics to calculate the efficiency. Experimental studies have been made of the dependence of luminescent efficiency on donor and acceptor concentration and this has been compared with a detailed treatment of the kinetics (Dapkus et al 1974). For p-type GaP the efficiency was found to increase linearly with excess acceptor concentration until the concentration reached $\sim 10^{18} \mathrm{~cm}^{-3}$, when the efficiency falls off. In the n-type material the fall off from the predicted dependence on excess donor concentration occurs at $10^{16}-10^{17} \mathrm{~cm}^{-3}$. Since these authors show that luminescent efficiency is dependent on the nitrogen concentration, as expected, these results can be understood in terms of the increasing internal electric fields. A difference in the behaviour

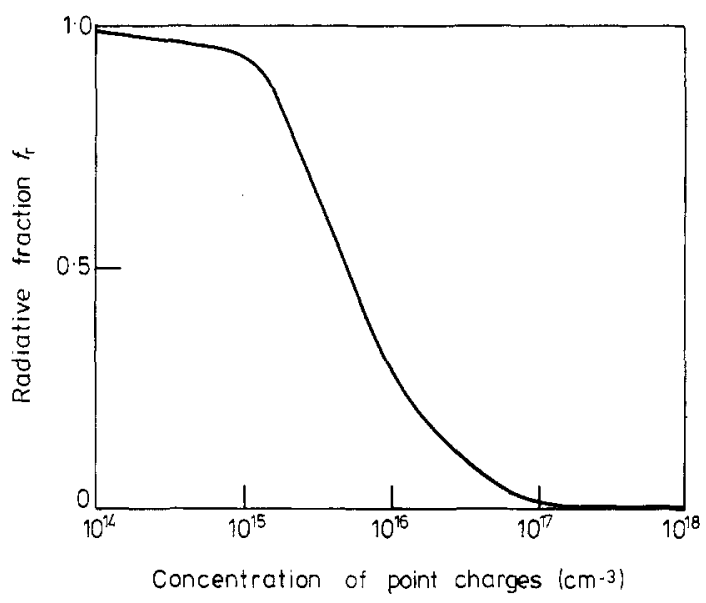

Figure 3. Radiative fraction of luminescent centres versus concentration of charged point defects. 
of $\mathrm{n}$ and $\mathrm{p}$-type materials is expected in this model since those nitrogen centres in high field regions in an n-type semiconductor are in regions of a higher than average density of positively charged donors whereas in a p-type material they are in a region of a higher than average density of negatively charged acceptors. Since the electron density will be lower near negatively charged species and higher in the vicinity of positively charged ones, in n-type semiconductors electrons will be attracted towards inactive nitrogen centres whereas in a p-type material they will be repelled towards active centres. Consequently, the decrease in radiative fraction will have higher than average effect on the efficiency in a n-type material and a lower than average effect in a p-type material as is observed.

The hole capture rate has had an insignificant effect on these results for the concentrations of defects $\sim 10^{14}-10^{18} \mathrm{~cm}^{-3}$. If a larger cross-section for the capture is used the effect will be even smaller, but at very low temperatures, and so low hole concentrations, the capture rate must become dominant. Under these conditions a small increase in temperature will create both more point charges, and hence internal electric fields, and more holes. The increased hole density will reduce the radiative lifetime and thus increase the efficiency. The increase in number point charges does not.become an important factor until it reaches a concentration of $\sim 10^{16} \mathrm{~cm}^{-3}$. As the hole capture rate depends on the total hole density, this argument is qualitatively the same for n-type, p-type and compensated semiconductors. The detailed temperature dependence is given not only by the ionisation of the dopants but also by the temperature dependence of the capture cross-section.

\section{Influence of dislocations}

The dislocations are considered as charged line defects as described in $\S 3$. They may have associated with them a charged atmosphere of point defects and the two extreme cases of no atmosphere, and thermal equilibrium have been considered. Figure 4 shows the radiative fraction of luminescent centres as a function of distance from the dislocation for two cases. In both cases there is a region around the dislocation with effectively no

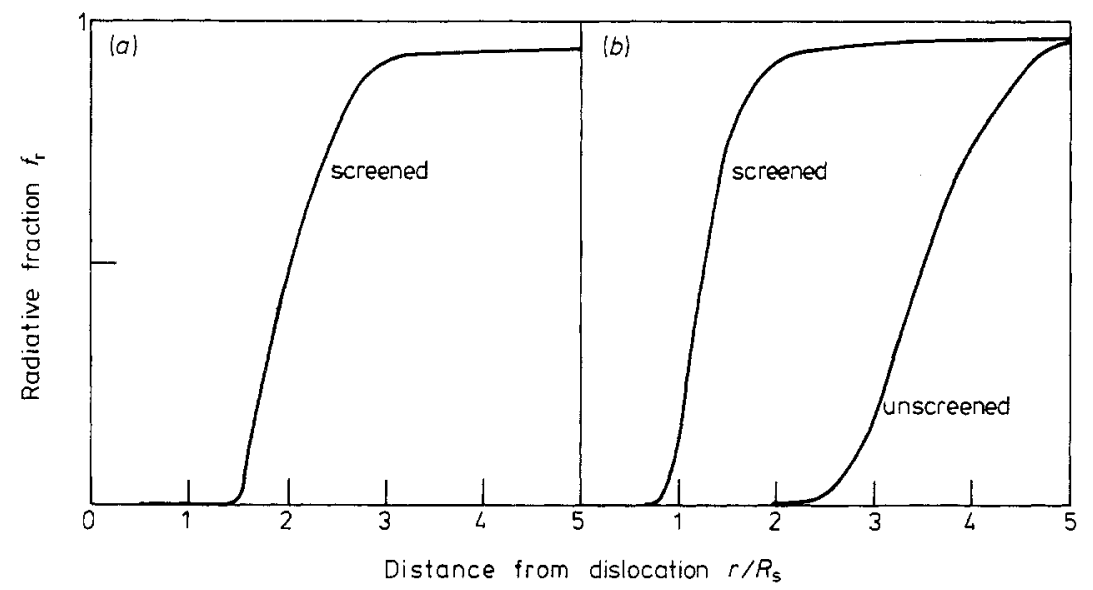

Figure 4. Radiative fraction of luminescent centres as a function of distance from dislocation (a) $\Delta E=0.01 \mathrm{eV}, \rho_{z}=10^{15} \mathrm{~cm}^{-3}$, and $R_{\mathrm{s}}=1617 \AA$ (b) $\Delta E=0.1 \mathrm{eV}, \rho_{z}=10^{17} \mathrm{~cm}^{-3}$, $R_{\mathrm{s}}=161.7 \AA$. In both cases the dislocation has 0.2 charges per $\AA$. 
luminescent centres, this will give rise to dark regions. In the first case the binding energy of the luminescent centre is $0.01 \mathrm{eV}$ (cf, $\mathrm{N}$ in $\mathrm{GaP}$ ). Only the result for the screened dislocation (i.e., with an atmosphere) is shown, the unscreened dislocation is much more damaging and the region of centre depletion extends out to $\sim 20 R_{\mathrm{s}}$ where $R_{\mathrm{s}}$ is the screening length. The distance scale is in units of $R_{\mathrm{s}}$, which in this case is $1617 \AA$ since we have assumed a concentration of charged point defects of $10^{15} \mathrm{~cm}^{-3}$. A charge of $0.2|e|$ per $\AA$ along the dislocation has been used. The second case shows the results for an electron binding energy of $0 \cdot 1 \mathrm{eV}$ and a concentration of point defects of $10^{17} \mathrm{~cm}^{-3}$. The deeper trap means that the effect of screened and unscreened dislocations is comparable. For both types the fields are less damaging than for the shallower centre, the screening length here is $162 \AA$.

It is clear that the electric fields associated with dislocations and their point charge atmosphere will lead to a depletion of active luminescent centres in the surrounding region of crystal. This may lead to a reduction in efficiency at high enough dislocation densities but will always produce dark regions in the material. The relative effect of screened and unscreeened dislocations depends on the electron binding energy and on the point charge density. Increasing the binding decreases the effect of the dislocation charge, and increasing the point charge density increases the effect of atmosphere around a screened dislocation. It is possible to construct circumstances where the unscreened dislocation is less damaging than the screened one. This treatment gives an interpretation of the dark regions observed around dislocations; the decrease in minority carrier lifetime with increasing dislocation density depends on the competing non-radiative recombination pathways that are not considered here.

\title{
6. Conclusions
}

The ionised donors and acceptors and charged dislocations cause internal electric fields in semiconductors, and these fields are sufficiently large to influence the luminescent properties of the material. They prevent the binding of electrons or holes to those luminescent centres which are in regions of the crystal where these fields are large. This produces two main effects. First, the effective concentration of luminescent centres decreases with increasing density of charged impurities above a density of $\sim 10^{16} \mathrm{~cm}^{-3}$. This causes a decrease in luminescent efficiency for high doping levels at reasonable temperatures. Secondly, the fields produce a spatial variation in the concentration of effective luminescent centres and, in particular, a depletion in the region around dislocations. This causes dark regions which may be observed in microscopic studies. These effects will be most marked for luminescent centres which only weakly bind the carriers, such as the nitrogen centre in gallium phosphide.

\section{References}

\author{
Abramowitz M and Stegun I A 1965 Handbook of Mathematical Functions (New York: Dover) \\ Bennett H S and Stoneham A M 1972 Phys. Rev. B6 3086 \\ Chandrasekhar S 1943 Rev. Mod. Phys. 151 \\ Cuthbert J D and Thomas D G 1967 Phys. Rev. 154763 \\ Czaja W 1971 Festkorp. X1 65 \\ Dapkus P D, Hackett, W H Lorimor O G and Bachrach R Z 1974 J. Appl. Phys, 454920 \\ Dean P J 1976 J. Lumin. 12/13 82
}


Duke C B 1969 Tunnelling in Solids (New York: Academic Press)

Eshelby J D, Newey W A, Pratt P L and Lidiard A B, 1958 Phil. Mag. 375

Faulkner R A 1968 Phys. Rev. 175991

Franz W 1952 Ann. Phys. 1117

Harding W R, Blenkinsop I D, and Wight D R 1976 Electron. Lett. 12503

Heinke W 1974 IOP Conf., Lattice Defects in Semiconductors ed F A Huntley (London: Institute of Physics) p. 380

Iwamoto M and Kasami A 1976 Appl. Phys. Lett. 28591

Kishino S, Chinone N, Nakashima H and Ito R 1976 Appl. Phys. Lett. 29488

Kuijpers F P J, Blok L and Vink A T 1975 J. Cryst. Growth 31165

Mims W B and Gillen R 1966 Phys. Rev. 148438

Moss T S, Burrell G J and Ellis B 1973 Semiconductor Opto-Electronics (London: Butterworth)

Neubert D 1974 IOP Conf., Lattice Defects in Semiconductors ed F A Huntley (London: Institute of Physics) p 398

Opdorp C van, Werkhoven C, and Vink A T 1977 Appl. Phys. Lett. 3040

Queisser H J 1976 Appl. Phys. 10275

Stoneham A M 1969 Rev. Mod. Phys. 4182

1974 J. Phys. C: Solid St. Phys. 7871 1975 Theory of Defects in Solids (Oxford: Clarendon Press)

Suzuki T and Matsumoto Y 1975 Appl. Phys. Lett. 26431

Thomas D G and Hopfield J J 1966 Phys. Rev. 150680

Titchmarsh J M, Booker G R, Harding W and Wight D R 1976 SERL Tech.J. 263.1

Werkhoven C, Opdorp C van and Vink A T 1976 Edinburgh Int. Symp. GaAs and Related Compounds, proceedings to be published

Whitworth R W 1972 Phys. Stat. Solidi B 54537

1975 Adv. Phys. 24203 